\title{
Cuidador familiar: sobrecarga e proteção
}

Ciro Augusto Floriani

Secretaria de Saúde de Estado do Rio de J aneiro. E-mail: cirofloriani@ terra.com.br

Abordaremos o cuidador do paciente com câncer avançado a partir de 3 premissas: 1) a de que maior parte dos cuidadores emerge do núcleo familiar; 2) sua atividade cotidiana de cuidados implica significativo ônus à sua vida; e, 3) há necessidade de que medidas de suporte a este cuidador considerem sua proteção como uma meta a ser perseguida.

A recente redefinição de cuidados paliativos da O rganização M undial de Saúde incorpora, além da já preconizada busca de qualidade de vida dos familiares do paciente com doença avançada, o suporte a estes familiares no período de enfrentamento da doença e, se necessário, durante a fase de luto. Portanto, para que este suporte seja adequadamente planejado e implementado, é condição necessária que inicialmente se conheça este importante ator que é o cuidador familiar. Assim, inicialmente veremos parte do que o estado da arte diz sobre ele. $\mathrm{Na}$ parte final, faremos algumas considerações com especial ênfase na necessidade de proteção a este cuidador.

0 que é um cuidador e quem é que desempenha este papel? A definição que aqui adotaremos é aquela apresentada pela Política Nacional de Saúde do Idoso², que diz que

"C uidador é a pessoa, membro ou não da família, que, com ou sem remuneração, cuida do idoso doente ou dependente no exercício das suas atividades diárias, tais como alimentação, higiene pessoal, medicação de rotina, acompanhamento aos serviços de saúde e demais serviços requeridos no cotidiano - como a ida a bancos ou farmácias -, excluídas as técnicas ou procedimentos identificados com profissões legalmente estabelecidas, particularmente na área da enfermagem".

A família costuma ser a principal origem do cuidador e as mulheres adultas e idosas preponderam nestes cuidados ${ }^{3}$. Porém, há também registros de cuidadores masculinos e de crianças e adolescentes ${ }^{4-6}$. Sabe-se, também, que algumas situações costumam determinar esta escolha: proximidade parental (esposas e filhas), proximidade física, proximidade afetiva e o fato de ser mulher?.

$\mathrm{H}$ á significativos estudos sobre o comportamento e as necessidades do cuidador no período de adoecimento do paciente com câncer, desde o diagnóstico, passando pelo tratamento inicial, recidivas da doença, retratamentos, sucessivas internações, até o encaminhamento para os cuidados paliativos - período estes que no seu conjunto tem sido chamado, na literatura, de "a jornada do câncer" -, e já se sabe que estas necessidades vão se diferenciando das do paciente, na medida em que este se encaminha para uma fase avançada e terminal de sua doença. Parte destes estudos reforçam a importância do cuidador ser percebido pelo médico já em período precoce desta "jornada", com o planejamento de intervenções de orientação, suporte e apoio $0^{6,8,9}$.

Sabe-se, também, que cuidar de um paciente com doença avançada no domicílio causa importante ônus ao cuidador e a sua família ${ }^{10-12}$, e, em relação ao cotidiano do cuidador, há uma literartura abundante de estudos que demonstram a sobrecarga que ele tem com sua estafante e estressora atividade de cuidados diários e ininterruptos. Talvez este seja um dos aspectos mais bem estudados e registrados com relação ao cuidador familiar, tanto no campo oncológico, quanto em outros campos dos cuidados em logo prazo. Estes custos ocorrem no nível físico, psíquico, social e financeiro e há estudos que demonstram, em circunstâncias específicas, um maior risco de infarto agudo do miocárdio e de morte para os cuidadores adultos e idosos ${ }^{13,14}$. Exclusão social, isolamento afetivo e social, depressão, erosão nos relacionamentos, perda da perspectiva de vida, distúrbios do sono, maior uso de psicotrópicos são alguns dos vários registros no contexto psicossocial do cuidador ${ }^{15}$. N ão encontramos estudos sobre síndrome de burnout em cuidadores familiares de pacientes com câncer avançado, mas sabe-se que 0 
burnout em cuidadores leva a práticas paternalistas4 e abusos e agressões ao paciente ${ }^{16}$.

Também erosão financeira do cuidador e da família tem sido descrita. 0 SUPPO RT ${ }^{17}$ mostrou que $20 \%$ dos cuidadores perderam seus empregos; $31 \%$ das famílias tiveram perda quase que total, ou total, das suas reservas financeiras; e $29 \%$ das famílias perderam a principal fonte de renda. N este estudo, as famílias mais atingidas foram as de baixa renda, com pacientes com menos de 45 anos e aquelas com pacientes com importante dependência. D escreveremos, sucintamente, al guns estudos realizados com cuidadores de pacientes oncológicos: (1) H ileman et al. ${ }^{18}$, estudando 492 cuidadores encontraram 6 categorias de necessidades: psicológica, informativa, relativa aos cuidados do paciente, pessoal, espiritual e relativa ao manejo do domicílio para provisão de cuidados; (2) Field eM cG aughey ${ }^{19}$ relatam pobre comunicação médica; tempo de consulta médica imprevisível; cuidados de enfermagem insuficientes e falta de conhecimento do cuidador sobre os cuidados do paciente; (3) 0 haeri et al. ${ }^{5}$, estudando 73 cuidadores de mulheres com câncer de mama avançado, encontram 3 aspectos estatisticamente significantes: pesado ônus familiar $(p=0,00001)$; ruptura com a rotina familiar $(p=0,00001)$ e pesado ônus financeiro para a família $(p=0,004)$; e (4) no estudo de Perez et al.20, feito com 230 pacientes com câncer avançado de colo de útero, o relato dos cuidadores e familiares foi de sofrimento, desgaste, sensação de impotência, sensação de abandono e desejo de morte, sendo detectado importantes distúrbios familiares e isolamento entre seus membros.

$M$ as há, igualmente, uma crescente literatura, principalmente dentro do campo da enfermagem, que tem demonstrado, por meio de investigação qualitativa, transformações no plano existencial para o cuidador, com resignificação de sua vida e com novas diretrizes e direcionamentos, frutos da vivência com os cuidados que realizaram ${ }^{4,9,21,22}$.

Em vista destes achados, várias propostas de intervenções têm sido sugeridas - abrangem medidas no plano físico, psicossocial e material/financeiro e na formação médica - e há autores, como Levine e Zuckerman ${ }^{12}$, que recomendam que os profissionais de saúde encarem a família como coadjuvantes nos cuidados e não como

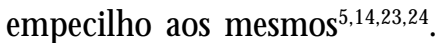

Em um aspecto parece haver concordância: a necessidade de ser instituído um processo de educação continuada para 0 cuidador, sem o qual medidas de cuidados implantadas tendem ao fracasso $25-28$.

Podemos também citar a recente modificação na legislação trabalhista francesa, com concessão de licença de 3 meses ao cuidador de uma pessoa com doença avançada, podendo esta atividade ser contabilizada como atividade laborativa ${ }^{29}$; ou a disposição do governo norte-americano, e de alguns estados deste país, em remunerar esta atividade ${ }^{30,31}$.

Assim sendo, o planejamento de estratégias que protejam o cuidador torna-se absolutamente fundamental, o que pode implicar para o gestor implementar medidas, entre outras, que viabilizem aos programas de cuidados paliativos, em casos bem selecionados, a absorção destes cuidados, provisória ou definitivamente nos casos, por exemplo, de ausência do cuidador.

Proteção é aqui entendida no sentido como é defendida pelos bioeticistas Schramm e Kottow - com a recém descrita bioética da proteção -, com medidas que dêem ao cuidador a possibilidade de planejar e de realizar outros interesses, com evitamento de um colapso em sua vida ${ }^{32}$.

Paradoxalmente, se já está estabelecido que a atividade de cuidar tende a erodir a vida do cuidador (ainda que haja relatos de tranfformações interiores), e que esta atividade deva, em última instância, visar o bem do paciente - que só pode ser atingida em sua plenitude se 0 cuidador estiver sem significativos agravos à sua saúde - por que as políticas de cuidados no fim da vida (e as de cuidados em longo prazo) depositam no cuidador uma expectativa tão grande, considerando-o fundamental para o sucesso destes programas? Por extensão, a partir de que momento histórico passa a se categorizar o cuidador e com que finalidade?

O utro aspecto a ser considerado é aquele relacionado às transformações na organização e na dinâmica da família contemporânea, e 0 quanto 0 câncer desestabiliza 0 paciente e sua família ${ }^{4,8,9}$. Até que ponto os programas de cuidados paliativos podem, de fato, contar com esta família e a partir de que momento as políticas de saúde podem e devem ajudar e, eventualmente, substituir o cuidador em suas funções?

$\mathrm{N}$ ão podemos deixar de citar aqui a intersecção dos cuidados paliativos com o campo do Atendimento D omiciliar (AD ) - $\mathrm{H}$ ome $\mathrm{C}$ are -, visto ser $\mathrm{o}$ atendimento no domicílio um dos vértices dos cuidados paliativos. D o modo como se tem desenvolvido o AD do tipo internação domiciliar (que é o modo de atendimento que pode suprir as necessidades do paciente com câncer avançado e durante a fase terminal no domicílio, dada a multiplicidade de problemas clínicos que este paciente apresenta nestas fases da doença), que tem sido organizado, por um lado, pela necessidade cada vez maior de otimização dos leitos hospitalares, devido aos altos custos financeiros com as 
internações prolongadas, gerando como conseqüência a ida para o domicílio do paciente quicker and sicker ${ }^{11}$ e, por outro lado, pelo modelo do "hospital sem paredes" ${ }^{33,34}$, com a organização de uma nova geografia dos cuidados à saúde em direção ao interior dos domicílios. As conseqüências deste modelo de cuidados paliativos no domicílio podem ser readmissões hospitalares, com aumento dos custos, sobrecarga ao cuidador e conflitos da família com a instituição, principalmente se a oferta de leitos for escassa - como costuma ocorrer - muitas vezes com intervenções judiciais. Aqui, deve-se investir em políticas de proteção aos mais vulneráveis, a saber, paciente e cuidador, ainda mais quando se sabe que a escolha para ser cuidador nem sempre é livre e muitas vezes feita sob pressão da instituição ${ }^{10,11,35}$. D eve-se ressaltar aqui que adesão do cuidador deve ser espontânea, estimulada e com suporte de informação, nunca imposta.

Tem sido relatado, também, o quanto o cuidado e a morte no domicílio marcam a vida dos familiares com relação ao que ocorre na casa, e que muitos membros da família não conseguem mais se desvencilhar das lembranças do que ocorreu no quarto em que ficou o paciente (cheiros, sons de monitores, organização do quarto para receber 0 paciente, etc) $)^{36}$.

Aliás, não tem sido possível boa prática de cuidados paliativos sem retaguarda hospitalar e disponibilidade de leitos, 24 horas por dia, 7 dias por semana ${ }^{37,38}$. M orrer em casa, ainda que desejado por muitos pacientes, não costuma ser a regra, mesmo em diferentes culturas; e, mesmo em países com uma boa rede de oferta de cuidados paliativos, os familiares muitas vezes preferem, na fase de cuidados terminais, levar o paciente para 0 hospital ${ }^{39}$. $\mathrm{N}$ esta fase terminal de cuidados os sintomas ficam mais intensos, o que aumenta a sobrecarga e 0 estresse do cuidador, tornando-o especialmente vulnerável nesta fase; neste momento, cuidador e sua família poderão não suportar ficar com o paciente agonizante em casa. Será, portanto, como preconizam alguns autores, que morrer em casa deva ser considerado um parâmetro de aferição da qualidade de um programa de cuidados paliativos e tradução de sua competência?

U m outro aspecto que é muito importante e que é impregnado de conflitos éticos diz respeito à omissão do diagnóstico e do prognóstico ao paciente com câncer incurável, aspecto este incompatível com a filosofia do moderno movimento hospice. M édico e família (e cuidador) muitas vezes pactuam pelo silêncio omisso, criando relações simétricas entre si e acentuando uma relação de assimetria com o paciente, isolando-o cada vez mais, com a construção de um cenário propício a práticas paternalistas, o que é diferente de um modelo de cuidados que leve em consideração decisões autônomas do paciente na busca de sua proteção e, por extensão, com possível salvaguarda de ônus adicional ao cuidador.

M esmo diante destas e de outras questões, e a despeito da sobrecarga imposta ao cuidador, inúmeros trabalhos enfatizam que boas práticas de cuidados paliativos trazem satisfação ao cuidador ${ }^{19,38,40-42}$. É necessário que em nosso contexto descubramos o que seja uma boa prática de cuidados paliativos. Cabe, neste sentido, que se realizem maiores estudos com respeito ao cuidador do paciente com câncer avançado em nosso meio, e que estes estudos levem em consideração a realidade sociocultural em que este cuidador vive, com uma focalização mais realista e com maior probabilidade de bons resultados, evitando-se, com isto, a importação de modelos de um modo descontextualizado. Torna-se, igualmente necessário que os aspectos bioéticos acerca do fim da vida sejam incluídos nestas pesquisas, levando em consideração a necessidade de proteger o cuidador, este importante ator social.

\section{R eferências}

1. World $\mathrm{H}$ ealth $\mathrm{O}$ rganization. $\mathrm{N}$ ational cancer control programmes: policies and managerial guidelines. 2nd ed. Geneva: WH 0 ; 2002 [cited 2003 M ay 3]. Available from: www.who.int/cancer

2. M inistério da Saúde. Portaria no.1395, de 13 de dezembro de 1999. Institui a Política N acional de Saúde do Idoso. Diário O ficial da República Federativa do Brasil (DF) dez 1999;(237-E) Seção 1:20.

3. Stone R, C afferata GL, Sangl J. Caregivers of the frail elderly: a national profile. Gerontologist. 1987;27(5):616-26.

4. Andershed B, Ternestedt BT. Involvement of relatives in care of the dying in different care cultures: development of a theoretical understanding. N urs Sci Q. 1999;12(1):45-51.

5. O haeri JU, Campbell OB, Ilesanmi AO, O migbodun AO. The psychosocial burden for some $\mathrm{N}$ igerian women with breast cancer and cervical cancer. Soc Sci Med. 1999;49:1541-9.

6. Soothill K, M orris SM , H arman JC, Francis B, M cillmurray M B. C ancer and faith. $\mathrm{H}$ aving faith - does it make a difference among patients and their informal carers? Scand J Caring Sci. 2002;16:256-63.

7. M endes PM T. Cuidadores: heróis anônimos do cotidiano [dissertação]. São Paulo (SP): Programa de Pós- 
Graduação em Serviço Social da Pontifícia Universidade Católica; 1995.

8. Thomas C, M orris SM . Informal carers in cancer contexts. Eur J Cancer Care. 2002;11:178-82.

9. Thomas $C, M$ orris $S M, H$ arman JC. Companions through cancer: the care given by informal carers in cancer contexts. Soc Sci Med. 2002;54:529-44.

10. Arras J, Dubler N N. Bringing the hospital home: ethical and social implications of high-tech home care. H astings Cent Rep. 1994;24(5):S19-28.

11. Collopy B, D ubler N, Zuckerman $C$. The ethics of home care: autonomy and accommodation. $\mathrm{H}$ astings $\mathrm{C}$ ent Rep. 1990;(Suppl):1-16.

12. Levine $\mathrm{C}$, Zuckerman $\mathrm{C}$. The trouble with families: toward an ethic of accommodation. Ann Intern M ed. 1999;130(2):148-52.

13. Lee S, Colditz GA, Berkman L, Kawachi I. Caregiving and the risk of coronary heart disease in U.S. women: a prospective study. Am J Prev M ed. 2003;24(2):113-9.

14. Schulz R, Beach SR. Caregiving as a risk factor for mortality: the caregiver health effects study. J Am M ed Assoc. 1999;282(23):2215-9.

15. Pitceathly $C, M$ aguire $P$. The psychological impact of cancer on patients' partners and other key relatives: a review. Eur J Cancer. 2003;39:1517-24.

16. Kayser-Jones J. H igh-Tech home care for elderly persons. In: Arras J, editor. Bringing the hospital home: ethical and social implications of high-tech home care. Baltimore: The Johns H opkins U niversity Press; 1995. p. 12945.

17. Covinsky KE, Goldman L, Cook F, O ye R, Desbiens N, Reding D, et al. The impact of serious illness on patients families. J Am M ed Assoc. 1994;272(23):1839-44.

18. H ileman JW, Lackey NR, H assanein RS. Identifying the needs of home caregivers of patients with cancer. O ncol N urs Forum. 1992;19(5):771-7.

19. Field D, M cgaughey J. An evaluation of palliative care services for cancer patients in the Southern $\mathrm{H}$ ealth and Social Services Board of Northern Ireland. Palliat Med. 1998;12:83-97.

20. Pérez AS, Chávez PF, D e La Lanza CC. Cuidados paliativos domiciliarios en pacientes com cáncer cervicouterino en etapas avanzadas. Rev Inst N ac Cancerol. 2000;46(1):10-6.

21. Caldas CP. 0 sentido do ser cuidando de uma pessoa idosa que vivencia um processo demencial [tese]. Rio de Janeiro: Escola de Enfermagem Anna N ery, Universidade Federal do Rio de Janeiro; 2000.

22. Koop PM, Strang VR. T he bereavement experience following home-based family caregiving for persons with advanced cancer. Clin Nurs Res. 2003;12(2):127-44.

23. Emanuel EJ, Fairclough D L, Slutsman J, Emanuel LL. Understanding economic and other burdens of terminal ilness: the experience of patients and their caregivers. Ann Intern M ed. 2000;132:451-9.

24. N eri AL. Bem-estar e estresse em familiares que cuidam de idosos fragilizados e de alta dependência. In: $N$ eri AL. Q ualidade de vida e idade madura. Campinas: Papirus; 2000. p. 237-85.

25. Caldas CP. A saúde do idoso: a arte de cuidar. Rio de Janeiro: Universidade Aberta da Terceira Idade, Universidade do Estado do Rio de Janeiro; 1998.

26. Dunlop R. Cancer: palliative care. London: Springer-Verlag; 1998.

27. Thielemann P. Educational needs of home caregivers of terminally ill patients: literature review. Am J H osp Palliat Care. 2000;17(4):253-7.

28. Adam J. Discharge planning of terminally ill patients home from an acute hospital. Int J Palliat Nurs. 2000;6(7):338-45.

29. Lei francesa de cuidados paliativos. Lei no. 99-477, de 9 de junho de 1999. M undo Saúde. 2003;27(1):191-4.

30. Bodenheimer T. Long-Term care for frail elderly people - the on lok model. N Engl J M ed. 1999;341(17):1324-8.

31. Levine $\mathrm{C}$. H ome sweet hospital: the nature and limits of private responsibilities for home health care. J Aging H ealth. 1999;11(3):341-59.

32. Schramm FR, Kottow M. Princípios bioéticos en salud pública: limitaciones y propuestas. Cad Saúde Pública. 2001;17(4):949-56.

33. Koren MJ. H ome care - who cares? N Engl J M ed. 1986;314(14):917-20.

34. Pousada L. High-Tech home care for elderly persons: what, why and how much? In: Arras J, editor. Bringing the hospital home: ethical and social implications of high-tech home care. Baltimore: The Johns Hopkins University Press; 1995. p. 107-28. 
35. M ontgomery RJV. The family role in the context of long-term care. J Aging H ealth. 1999;11(3):383-416.

36. Alvarez AM. Tendo que cuidar: a vivência do idoso e de sua família cuidadora no processo de cuidar e ser cuidado em contexto domiciliar [tese]. Florianópolis (SC): Escola de Enfermagem da Universidade Federal de Santa Catarina; 2001.

37. D oyle D, Jeffrey D. Palliative care in the home. Oxford: O xford University Press; 2000.

38. Steinhauser KE, Christakis N A, Clipp EC, M cneilly M, M cintyre L, Tulsky JA. Factors considered important at the end of life by patients, family, physicians, and other care providers. J Am M ed Assoc. 2000;284(19):2476-82.

39. Procter S, Wilcockson J, Pearson P, Allgar V. Going home from hospital: the carer / patient dyad. J Adv N urs. 2001;35(2):206-17.

40. Rollison $B, C$ arlsson $M$. Evaluation of advanced home care (AH C). The next-of-kin's experiences. Eur J O ncol N urs. 2002;6(2):100-6.

41. Steele LL, M ills B, Long M R, H agopian GA. Patient and caregiver satisfaction with end-of-life care: does high satisfaction mean high quality of care? Am J H osp Palliat Care. 2002;19(1):19-27.

42. Wennman-Larsen A, Tieshelman C. Advanced home care for cancer patients at the end of life: a qualitative study of hopes and expectations of family caregivers. Scand J Caring Sci. 2002;16:240-7. 